\title{
Análise dos Pólipos Colorretais em 3.491 Videocolonoscopias
}

\author{
Analysis of Colorrectal Polyps in 3.491 Videocolonoscopies
}

\author{
JOSÉMAURO DOS SANTOS ${ }^{1}$; FELIPEFELÍCIO²; HUMBERTO FENNER LYRAJUNIOR ${ }^{3}$; \\ MARIAROBERTACARDOSO MARTINS ${ }^{4}$; FERNÃOBITTENCOURTCARDOSO ${ }^{5}$
}

\author{
${ }^{1}$ Doutor em Medicina e Professor Adjunto do Departamento de Clínica Cirúrgica da Universidade Federal \\ de Santa Catarina; ${ }^{2}$ Professor Titular do Departamento de Clínica Cirúrgica da Universidade Federal de Santa \\ Catarina: ${ }^{3}$ Membro do Serviço de Coloproctologia do Hospital Universitário da Universidade Federal de Santa \\ Catarina; ${ }^{4}$ Graduação em Medicina pela Universidade Federal de Santa Catarina; ${ }^{5}$ Acadêmico do quinto ano do curso \\ de Medicina da Universidade Federal de Santa Catarina - Santa Catarina - Brasil - Santa Catarina - Brasil.
}

\begin{abstract}
SANTOS JM; FELÍCIO F; LYRA JUNIOR HF; MARTINS MRC; CARDOSO FB. Análise dos Pólipos Colorretais em 3.491 Videocolonoscopias. Rev bras Coloproct, 2008;28(3): 299-305.

RESUMO: Introdução: A ressecção dos pólipos do intestino grosso é uma medida importante na diminuição da incidência do câncer colorretal. $O$ objetivo do presente estudo é verificar a incidência dos pólipos colorretais, seu tipo histológico, o índice de malignização e de ocorrência de novas lesões nos pacientes submetidos a exame colonoscópico por indicações diversas no Serviço de Coloproctologia do Hospital de Caridade de Florianópolis - SC. Método: Realizado estudo retrospectivo de 3491 pacientes submetidos à videocolonoscopia no período de janeiro de 1992 a outubro de 2006. Foi avaliada a incidência dos pólipos colorretais de acordo com sexo e idade, tipo histológico e a presença de adenocarcinoma. Foram excluídos deste estudo as síndromes polipóides genéticas e os pólipos não ressecados ou com exames anatomopatológicos incompletos ou extraviados. Resultados: Dos 3491 exames realizados, foram encontrados pólipos em 1046 (29,96\%), totalizando 1899 lesões em 865 pacientes. Destes $53,94 \%$ eram do sexo masculino, $46,06 \%$ feminino e $58,46 \%$ tinham mais de 60 anos. Foram avaliados 1579 pólipos colorretais, cujo estudo histopatológico mostrou 31,54\% adenomas tubulares, 14,19\% adenomas túbulo-vilosos, 1,65\% adenomas vilosos, 36,03\% pólipos hiperplásicos, $7,85 \%$ pólipos inflamatórios e $7,85 \%$ de outros tipos histológicos. Foram observados 24 (1,52\%) adenocarcinomas polipóides e $18(1,14 \%)$ adenomas com focos de adenocarcinoma. Conclusão: A incidência de pólipos nos pacientes estudados foi bastante alta, sendo os adenomas o tipo histológico mais freqüente, com risco de malignização de 1,14\% e os adenocarcinomas polipóides $\mathbf{1 , 5 2 \%}$. Os pacientes portadores de adenomas colorretais devem ser submetidos a exames de controle, devido ao alto índice de ocorrência de novas lesões.
\end{abstract}

Descritores: Pólipos, Colonoscopia, Câncer Colorretal.

\section{INTRODUÇÃO}

O câncer colorretal é causa importante de morbi-mortalidade em todo o mundo. Nos Estados Unidos, 73182 homens e 70763 mulheres tiveram o diagnóstico de câncer colorretal em 2003, resultando em 27990 e 27793 mortes decorrentes dessa doença em homens e mulheres, respectivamente, neste mesmo ano $^{1}$. No Brasil, foram notificados 11390 novos casos em homens e 13970 em mulheres no ano de 2006, ficando o câncer colorretal como o quinto tumor malig- no mais freqüente em homens e o quarto em mulheres. A maior incidência desta doença encontra-se entre 50 e 70 anos, mas o risco de desenvolvimento encontra-se aumentado a partir dos 40 anos $^{2}$. Com o diagnóstico precoce através do exame colonoscópico e posterior ressecção ainda em fase de adenoma, a sequiência adenoma-câncer pode ser interrompida, diminuindo a incidência do câncer colorretal ${ }^{3,4,5,6-10}$. Com as técnicas modernas, a maioria dos pólipos podem ser removidos através da colonoscopia com um risco pequeno para o paciente. Os pólipos pediculados podem ser res-

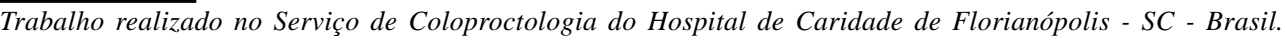

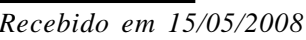

Aceito para publicação em 12/06/2008 
secados através da alça de diatermia introduzida através do colonoscópio e conectada a um bisturi elétrico. Os pólipos sésseis podem ser retirados em fatias, pelo mesmo método ou pela técnica de mucosectomia, por um endoscopista experiente, uma vez que os riscos de complicações são maiores nesses casos. As principais complicações com o método são a perfuração intestinal e o sangramento, com incidências baixas e dependentes da experiência do endoscopista ${ }^{11,12,13}$. Após a ressecção dos adenomas, há um risco de ocorrência de novas lesões estimado entre 32,0 a 41,7\%, de acordo com o National Polyp Study ${ }^{13}$; sendo, portanto, necessário um seguimento desses pacientes pelo menos até 3 anos após a polipectomia. Caso não sejam encontrados novos pólipos, esse tempo estende-se para 5 anos. Em caso de presença de adenocarcinoma, fazse necessário um seguimento mais rigoroso em 6 meses, 2 e 5 anos, dependendo do nível de invasão do mesmo ${ }^{14,15,16}$. O objetivo do presente estudo é analisar a incidência dos pólipos, seu tipo histológico e o índice de malignização nos pacientes submetidos a exame videocolonoscópico por indicações diversas, bem como a necessidade de exames endoscópicos de controle nesses pacientes.

\section{MÉTODO}

Estudo retrospectivo transversal observacional, com todos os pacientes submetidos à colonoscopia no Serviço de Coloproctologia do Hospital de Caridade Irmandade Senhor Jesus dos Passos de Florianópolis SC, no período compreendido entre janeiro de 1992 a outubro de 2006.

Foram analisados todos os exames onde foram diagnosticados pólipos colorretais, seu percentual em relação às colonoscopias avaliadas e o número de pacientes com a doença. Os pacientes foram divididos por sexo e faixa etária (até 20, 21 a 30, 31 a 40, 41 a 50,51 a 60 e mais de 60 anos). Além disso, estudou-se todas as indicações dos exames colonoscópicos. Os pólipos foram analisados de acordo com seu tamanho (menor que $10 \mathrm{~mm}$ de diâmetro ou maior ou igual a 10 $\mathrm{mm}$ ), base (séssil ou pediculada), tipo histológico (adenoma tubular, adenoma viloso, adenoma tubuloviloso, pólipo hiperplásico, pólipo inflamatório, adenocarcinoma ou outros), presença de atipias (leves, moderadas ou acentuadas) e focos de adenocarcinoma. Foi também realizada a análise dos exames colonoscópicos de seguimento desses pacien- tes e o índice de ocorrência de novos pólipos.

Foram excluídos deste estudo as síndromes polipóides genéticas, os pólipos não ressecados ou cujo anatomopatológico estava incompleto ou foi extraviado.

Os dados foram coletados em um protocolo padrão estabelecido para a pesquisa e armazenados no programa EpiData 3.1.

Para análise estatística utilizou-se o programa EpiData Analysis V1.1, sendo considerado estatisticamente significativo $p=0,05$.

No dia anterior ao exame, os pacientes realizaram preparo do cólon com dieta líquida sem resíduos e $30 \mathrm{~g}$ de sulfato de sódio por via oral. No dia do exame, receberam manitol a $10 \%$ via oral antes do início do mesmo. Foi realizada a sedação endovenosa com $50 \mathrm{mg}$ de meperidina e 7,5 mg de midazolan. Para reversão da sedação foi utilizado naloxona e, eventualmente, o flumazenil após o procedimento.

O videocolonoscópio utilizado foi o Pentax EC3830FK.

\section{RESULTADOS}

Foram analisados 3491 videocolonoscopias, sendo 3375 destas realizados por um único examinador. Dentre os exames realizados, em 1046 (29,96\%) foram encontrados pólipos colorretais num total de 865 pacientes, e em $58,13 \%$ destes a lesão polipóide era única.

Dos 865 pacientes examinados, 53,94\% eram do sexo masculino e $46,06 \%$ do sexo feminino. A idade média foi de 62,42 anos, com extremos de 4 e 94 anos e uma mediana de 63 anos. Dos pacientes avaliados $58,13 \%$ tinham mais de 60 anos (Tabela 1 ).

Tabela 1 - Distribuição dos pacientes por faixa etária.

\begin{tabular}{lrc}
\hline Faixa Etária & $\mathbf{N}^{\mathbf{0}}$ & \% \\
\hline Até 20 anos & 9 & 0,86 \\
21 a 30 anos & 12 & 1,15 \\
31 a 40 anos & 50 & 4,78 \\
41 a 50 anos & 128 & 12,24 \\
51 a 60 anos & 233 & 22,27 \\
Mais de 60 anos & 608 & 58,13 \\
Desconhecida & 6 & 0,57 \\
Total & 1046 & 100 \\
\hline
\end{tabular}


As indicações mais freqüentes para a realização da colonoscopia foram sangramento intestinal $(28,49 \%)$, dor abdominal $(21,03 \%)$, seguimento de polipectomia $(20,36 \%)$, diarréia $(13,96 \%)$, constipação intestinal $(12,33 \%)$ e alteração do hábito intestinal $(11,76 \%)$ (Tabela 2). A indicação de sangramento intestinal inclui enterorragia, hematoquesia, hemorragia digestiva baixa, melena, sangramento retal e pesquisa de sangue oculto nas fezes positiva. Muitos pacientes apresentaram mais de uma indicação.

A localização mais freqüente dos pólipos foi no cólon ascendente com $452(23,80 \%)$ lesões, seguida de 408 (21,48\%) no cólon sigmóide, $325(17,11 \%)$ no cólon transverso e $302(15,90 \%)$ no reto (Tabela 3$)$.

Dos pólipos avaliados, $86,82 \%$ apresentavam menos que 10 milímetros de diâmetro, enquanto $13,18 \%$ tinham 10 milímetros ou mais. Dentre esses, 34 pólipos sésseis foram ressecados pela técnica de mucosectomia. Em relação à base, observou-se que $81,09 \%$ eram sésseis e $18,91 \%$ pediculados.

Com a exclusão dos pólipos não ressecados, restaram 1899 lesões, sendo que dessas, 320 tiveram laudo anatomopatológico incompleto ou foram extraviados durante o exame, finalizando 1579 pólipos colorretais a serem analisados.

Dos 1579 pólipos colorretais analisados, o estudo histopatológico mostrou que $31,54 \%$ eram adenomas tubulares, $14,19 \%$ adenomas túbulo-vilosos,
1,65\% adenomas vilosos, 36,03\% pólipos hiperplásicos, $7,22 \%$ pólipos inflamatórios, 7,85\% outros tipos histológicos, incluindo lipomas, hemangiomas, leiomiomas submucosos, hamartomas, pseudopólipos, hiperplasias linfóides e um caso de adenoma serrilhado (Tabela 4). Foram observados 24 (1,52\%) adenocarcinomas polipóides e $18(1,14 \%)$ adenomas com focos de adenocarcinoma, totalizando uma incidência de 2,66\% de adenocarcinoma dentre os pólipos estudados. Destes, 7 pacientes foram submetidos à ressecção cirúrgica.

Dos 18 pólipos com focos de adenocarcinoma encontrados, $8(1,61 \%)$ eram adenomas tubulares, 6 $(2,68 \%)$ adenomas tubulovilosos e $4(15,38 \%)$ adenomas vilosos.

Foram realizados 117 exames para seguimento dos pacientes após 1 ano da polipectomia, sendo que $55(47,01 \%)$ deles apresentaram ocorrência de novos pólipos, e $62(52,99 \%)$ não apresentaram. O número de exames para seguimento diminuiu gradativamente a cada ano por falta de aderência dos pacientes ao tratamento, permanecendo o número de ocorrência de novas lesões em pouco menos da metade dos pacientes nos anos subseqüentes.

O grau de atipias dos adenomas foi descrito em $500(66,84 \%)$ das 748 lesões encontradas, sendo atipias leves em 290 (58\%), atipias moderadas em 184 $(36,8 \%)$ e atipias acentuadas em $26(5,2 \%)$ deles.

Tabela 2 - Indicações de videocolonoscopia.

\begin{tabular}{lcc}
\hline Indicações & $\mathbf{N}^{\mathbf{0}}$ & $\mathbf{\%}$ \\
\hline Sangramento intestinal & 298 & 28,49 \\
Dor abdominal & 220 & 21,03 \\
Seguimento pós-polipectomia & 213 & 20,36 \\
Diarréia & 146 & 13,96 \\
Constipação intestinal & 129 & 12,33 \\
Alteração do hábito intestinal & 123 & 11,76 \\
Emagrecimento & 81 & 7,74 \\
Anemia & 70 & 6,69 \\
Pólipo à retossigmoidoscopia & 67 & 6,40 \\
Seguimento pós-operatório de câncer intestinal & 65 & 6,21 \\
História familiar de neoplasia intestinal & 59 & 5,64 \\
Ressecção intestinal anterior & 26 & 2,48 \\
Seguimento de DDC & 24 & 2,29 \\
Seguimento de outras neoplasias & 23 & 2,20 \\
Mucorréia & 22 & 2,10 \\
\hline
\end{tabular}


Tabela 3 - Localização dos pólipos no intestino grosso.

\begin{tabular}{lrc}
\hline Localização & $\mathbf{N}^{\mathbf{0}}$ & $\mathbf{\%}$ \\
\hline Ascendente & 452 & 23,80 \\
Sigmóide & 408 & 21,48 \\
Transverso & 325 & 17,11 \\
Reto & 302 & 15,90 \\
Ceco & 184 & 9,69 \\
Descendente & 158 & 8,32 \\
Retossigmóide & 49 & 2,58 \\
Íleo terminal & 04 & 0,21 \\
Difusos & 17 & 0,90 \\
Total & 1899 & 100 \\
\hline
\end{tabular}

Tabela 4 - Tipo histológico dos pólipos analisados.

\begin{tabular}{lrr}
\hline Tipo Histológico & $\mathbf{N}^{\mathbf{0}}$ & $\mathbf{\%}$ \\
\hline Adenoma Tubular & 498 & 31,54 \\
Adenoma Tubuloviloso & 224 & 14,19 \\
Adenoma Viloso & 26 & 1,65 \\
Pólipo Hiperplásico & 569 & 36,03 \\
Pólipo Inflamatório & 114 & 7,22 \\
Adenocarcinoma & 24 & 1,52 \\
Outros & 124 & 7,85 \\
Total & 1579 & 100 \\
\hline
\end{tabular}

\section{DISCUSSÃO}

Pólipos colorretais são achados freqüentes nas colonoscopias, incidindo em mais de $25 \%$ dos exames realizados segundo Giacosa et al.. ${ }^{15}$ Bonadeo et al. ${ }^{17}$, numa série de 2068 colonoscopias, encontraram pólipos em 19,8\% dos exames. Já Winawer e Markowitz ${ }^{18}$ relataram incidências de até $41 \%$ em estudos em autópsias, e outros estudos demonstraram taxas em torno de $20 \%^{12}$. No presente trabalho a incidência dos pólipos colorretais foi de $29,96 \%$.

Winawer e Markowitz ${ }^{18}$ descreveram taxas de incidência de adenomas de 21 a $28 \%$ em pacientes entre 50 e 59 anos, 41 a $45 \%$ entre 60 e 69 anos e 53 a $58 \%$ em pacientes com mais de 70 anos. Bellegooijen et al. ${ }^{19}$ demonstraram uma idade média dos pacientes portadores de pólipos de 62,1 anos, com
53\% dos pacientes sendo do sexo masculino e $47 \%$ feminino. A média de idade dos pacientes portadores de pólipos no presente estudo foi de 62,42 anos, estando de acordo com a literatura. A incidência por sexo, que neste estudo mostrou valores de $53,94 \%$ no sexo masculino e $46,06 \%$, no sexo feminino, também está de acordo com a literatura ${ }^{12,9,20}$.

As indicações para a realização das colonoscopias relatadas nos estudos pesquisados são semelhantes às do presente estudo, como sangramento retal, seguimento de polipectomia e câncer, alterações de hábito intestinal, história familiar de neoplasia. As indicações não aparecem necessariamente na mesma ordem de freqüência, o que pode ser explicado pela coexistência de outras doenças e pelas indicações múltiplas, o que dificulta saber se a queixa é decorrente ou não do pólipo ${ }^{12,20}$.

Na série de 311 casos de Church ${ }^{20}$, em que $68,2 \%$ dos pólipos encontravam-se no cólon direito e $31,8 \%$ no cólon esquerdo, 65 pólipos estavam localizados no ceco, 16 na válvula ileocecal, 69 no cólon ascendente, 20 no ângulo hepático do cólon, 31 no cólon transverso, 11 no ângulo esplênico, 21 no cólon descendente e 75 no cólon sigmóide. Neste estudo,

os locais de maior incidência dos pólipos foram os cólons ascendente $(23,80 \%)$ e sigmóide $(21,48 \%)$, seguidos do transverso $(17,11 \%)$ e reto $(15,90 \%)$, dados esses semelhantes aos encontrados na literatura ${ }^{12,18}$.

Os adenomas colorretais possuem um importante significado clínico devido ao seu potencial de malignização, sendo já bem estabelecida a existência de uma seqüência adenoma-câncer. O National Polyp Study demonstrou uma redução de 76 a $90 \%$ na incidência de câncer colorretal nos pacientes submetidos à ressecção endoscópica dos adenomas colorretais e posterior exames de controle, em comparação com grupos controle; comprovando assim, a diminuição do risco de desenvolvimento de câncer colorretal com a ressecção colonoscópica dos adenomas ${ }^{13-22,18,23}$.

Os adenomas podem ser classificados histologicamente em adenoma tubular, adenoma tubuloviloso e adenoma viloso. De acordo com a classificação da World Health Organization, os adenomas tubulares contêm de 0 a $25 \%$ de componente viloso, os adenomas tubulovilosos de 25 a $75 \%$ e os adenomas vilosos contêm de 75 a $100 \%$ de tecido viloso ${ }^{24}$. O tipo tubular é o adenoma mais comumente encontrado (70 a $85 \%$ ), sendo seguido pelo tipo tubuloviloso (25\%) e viloso (5\%), valores esses semelhantes aos encontra- 
dos no presente estudo, onde o adenoma tubular foi verificado em $66,58 \%$, o tubuloviloso em $29,95 \%$ e o viloso em $3,47 \%$ de todos os adenomas encontrados. Dentre todos os tipos de pólipos estudados, ou seja, 1579 lesões, a incidência dos adenomas tubulares, tubulovilosos e vilosos foi de, respectivamente, $31,54 \%$, $14,19 \%$ e $1,65 \%{ }^{3,18,24}$.

Os adenomas vilosos possuem um maior potencial de malignização, o que pôde ser comprovado neste estudo, onde $15,38 \%$ deles apresentaram focos de adenocarcinoma 12,9,24. Winawer e Markowitz ${ }^{18}$ relataram uma taxa de $40,7 \%$ de malignização nesse tipo de adenoma.

Algum grau de atipia pode ser encontrado em todos os adenomas, podendo ser leves, moderadas ou acentuadas, e o potencial de malignização aumenta quanto mais acentuado for esse grau ${ }^{29}$. De acordo com o National Polyp Study ${ }^{13}, 86 \%$ dos adenomas apresentam atipias leves, $8 \%$ moderadas e $6 \%$ acentuadas, também conhecidas como carcinoma in situ. Neste estudo, $58 \%$ dos adenomas apresentavam atipias leves, 36,8\% moderadas e 5,2\% tinham atipias acentuadas. Essa diferença de valores pode ser explicada, em parte, pelo fato de que o National Polyp Study analisou apenas pólipos maiores ou iguais a $1 \mathrm{~cm}$ de diâmetro, enquanto esse estudo não fez restrições de tamanho. Outro fato que também contribuiu para a diferença nesses percentuais é a subjetividade dessas alterações, que é patologista-dependente ${ }^{13,18,24}$.

A incidência de adenomas é, sem dúvidas, muito maior que a incidência de câncer colorretal. Giacosa et al. ${ }^{15}$ acredita que menos de $1 \%$, evolui para adenocarcinoma. Não é possível determinar quais adenomas evoluirão para câncer, todavia alguns fatores estão ligados a um maior risco de malignização, como histologia vilosa, tamanho maior das lesões e presença de atipias acentuadas 6,9,18.

Seitz et al. ${ }^{25}$ descreveram uma variação na taxa de malignização entre 2 e 9,4\%, taxa essa descrita por Bond ${ }^{24}$ entre 4 e $7 \%$ e por Schmiegel et al. ${ }^{14}$ de $5 \%$. Ballegooijen et al. ${ }^{19}$ comprovaram que pacientes portadores de adenomas apresentavam de 0,2 a 1,3 vezes mais chances de desenvolver câncer colorretal. Neste estudo, foram observados 24 (1,52\%) adenocarcinomas polipóides e 18 (1,14\%) adenomas com focos de adenocarcinomas, totalizando $2,66 \%$ de câncer nos pólipos analisados. Além dos pacientes com adenocarcinomas polipóides, foi realizado tratamento cirúrgico em 7 pacientes com adenomas com focos de adenocarcinomas invadindo a submucosa, sendo que um deles recusou a cirurgia pelos riscos em decorrência da idade avançada, e decidiu realizar apenas exames de acompanhamento. Este paciente apresentava 2 adenomas de reto com focos de adenocarcinoma invadindo a submucosa que foram ressecados, e após 2 anos apresentou lesão metastática ganglionar no mesorreto alto, sendo submetido à ressecção cirúrgica com boa evolução.

Os pólipos hiperplásicos podem ser definidos como lesões causadas pela inibição da morte celular programada ou apoptose e, juntamente com os adenomas, são os pólipos mais comumente encontrados durante o exame colonoscópico, o que também pôde ser observado neste estudo, já que de todas as lesões analisadas, 36,03\% eram hiperplásicas ${ }^{18,26,27}$. Até recentemente, os pólipos hiperplásicos eram considerados lesões sem nenhum potencial de malignização, conceito esse que tem sido questionado nos últimos anos com o reconhecimento recente do adenoma serrilhado 26, 27. Reconhecido há pouco tempo, o adenoma serrilhado pode ser caracterizado pela associação de tecido adenomatoso e hiperplásico, o que nos faz repensar a respeito da necessidade de investigação nos portadores de pólipos hiperplásicos, pois assim como os adenomas, há evidências de que os mesmos podem estar associados à neoplasia colorretal ${ }^{3,27,28}$. Neste estudo, foi encontrado apenas um caso de adenoma serrilhado.

O seguimento colonoscópico é recomendado a todos os pacientes portadores de adenomas. De acordo com o National Polyp Study ${ }^{13}$, o intervalo seguro para a realização de uma nova colonoscopia para seguimento pós-polipectomia é de 3 anos. Este estudo avaliou 1418 pacientes, e comparou um grupo que realizou o seguimento após 1 ano e após 3 anos, e outro grupo apenas após 3 anos, sem diferença significativa entre os dois grupos ${ }^{29,13,18,30}$. Em alguns casos, o exame de controle deve ser realizado mais precocemente, dentro de 3 a 6 meses ou 1 ano, a fim de garantir uma maior segurança ao paciente, como em casos de ressecção duvidosa da lesão, pólipos sésseis grandes, ressecção de múltiplos adenomas ou exame do restante do cólon prejudicado por preparo insuficiente do intestino ${ }^{6,22}$.

No presente estudo foi observado que, 117 pessoas realizaram o exame para seguimento no primeiro ano após a polipectomia, 75 no segundo ano e 57 no terceiro, com um achado de novo pólipo em quase 
metade desses exames. $\mathrm{O}$ pequeno número de exames de seguimento pode ser explicado, em parte, pelo baixo grau de aderência dos pacientes aos programas de rastreamento, ou por terem realizado exames em outros serviços. Há, portanto, uma necessidade de realização de mais estudos a fim de confirmar, de maneira mais adequada, o intervalo de tempo ideal para a realização de novas colonoscopias nos pacientes submetidos a polipectomias.

Segundo Winawer et al. ${ }^{13}$, um adenoma leva em torno de 5 anos para se desenvolver a partir da mucosa normal do intestino, e em torno de 10 anos para transformar-se em câncer; sendo, portanto, a realização da videocolonoscopia uma maneira segura de diagnosticar e tratar essas lesões enquanto elas ainda são benignas e facilmente ressecáveis ${ }^{23-25,31,32}$.

Hemorragia, perfuração intestinal, polipectomia inadequada e o não reconhecimento de um câncer são complicações e dificuldades que podem ocorrer na colonoscopia, com prevalências descritas por Church ${ }^{20}$ variando de 1 a 9\%, dependendo da experiência do examinador ${ }^{12,6,9,18,20,33}$. Winawer e Markowitz ${ }^{18}$ descreveram taxas de perfuração ou grandes sangramentos de 0,1 a $0,2 \%$ dos exames, Waye et al ${ }^{9}$ relataram índices de menos de $1 \%$ de perfuração e 0,27 a 2,21\% de sangramento, enquanto Repici e Tricerri ${ }^{33}$ apontaram sangramento em 0,3 a $6,1 \%$ das colonoscopias. $\mathrm{Na}$ presente amostra, foram observados $5(0,48 \%)$ casos de perfuração intestinal que foram tratados cirurgicamente, e $2(0,19 \%)$ casos de sangramento póspolipectomia, necessitando de internação hospitalar, mas com resolução espontânea. Não houve óbitos no presente estudo.

Com base nesses dados, pode-se afirmar que a incidência de pólipos nos pacientes submetidos à colonoscopia foi de $29,96 \%$, sendo os adenomas o tipo histológico mais freqüente, com risco de malignização de $1,14 \%$ e adenocarcinomas polipóides de $1,52 \%$. Os pacientes portadores de adenomas colorretais ressecados devem ser submetidos a exame de controle em até 3 anos após a polipectomia, devido ao alto índice de ocorrência de novas lesões.

\begin{abstract}
Introduction: The resection of colorectal polyps is an important role in decreasing the incidence of colorectal cancer. The objective of this study is to determine the incidence of polyps, histological type, malignant transformation rate and the appearance of new polyps in patients that underwent videocolonoscopy for a wide range of indications in the coloproctology service of the Caridade Hospital in Florianópolis - SC. Method: Retrospective analysis of 3,491 patients submitted to videocolonoscopy from January 2002 to October 2006. The incidence of colorectal polyps by sex and age, histological types and the presence of adenocarcinoma were determined. Familial polypoid syndromes, unresected polyps and patients whose pathology records were incomplete or had been lost were excluded. Results: Polyps were found in 1,046 (29.96\%) out of 3,491 videocolonoscopies, totaling 1,899 polyps in 865 patients. $53.94 \%$ of patients were male and $46.06 \%$ female, and $58.46 \%$ of them aged over 60 years. The histological analysis of $\mathbf{1 , 5 7 9}$ colorectal polyps showed that $31.54 \%$ were tubular adenomas, $14.19 \%$ tubulovillous adenomas, $1.65 \%$ villous adenomas, $36.03 \%$ hyperplasic polyps, $7.22 \%$ inflammatory polyps and $7.85 \%$ of other histological types. 24 (1.52\%) polypoid adenocarcinomas and $18(1.14 \%)$ adenocarcinoma focci in adenomas were observed. Conclusion: The incidence of polyps in the studied sample was high, with adenoma being the most frequent histological type, with risk of malignization of $1.14 \%$ and polypoid adenocarcinomas in $\mathbf{1 . 5 2 \%}$. Patients with colorectal adenomas must be followed-up due to the increased likelihood of the appearance of new lesions.
\end{abstract}

Keywords: Polyps, Colonoscopy, Colorectal Cancer.

\section{REFERÊNCIAS}

1. Centers of Disease Control and Prevention [homepage na internet]. Atlanta: Department of Health and Human Services [atualizada em 2007 May 16; acesso em 2007 May 19]. Disponível em: http://www.cdc.gov/

2. Instituto Nacional de Câncer [homepage na internet]. Brasil: Ministério da Saúde c1996-2007 [atualizada em 2007 May 16; acesso em 2007 May 19]. Disponível em: http:// www.inca.gov.br/
3. Lyra Jr HF, Bonardi MA, Schiochet VNC, Baldin Jr A, Carmes ER, Sartor MC, Pisani JC, Brenner S, Bornardi RA. Importância da colonoscopia no rastreamento de pólipos e câncer em pacientes portadores de pólipos retais. Rev Bras Coloproct. 2005;25(3):226-34.

4. Winawer SJ. The achievements, impact, and future of the National Polyp Study. Gastrointest Endosc. 2006 Dec;64(6):975-8.

5. Lewis JD, Ng K, Hung KE, Bilker WB, Berlin JA, Brensinger $\mathrm{C}$, et al. Detection of proximal adenomatous polyps with 
screening sigmoidoscopy: a systematic review and metaanalysis of screening colonoscopy. Arch Intern Med. 2003 Feb 24;163(4):413-20.

6. Miller K, Waye JD. Colorectal polyps in the elderly: what should be done? Drugs Aging. 2002;19(6):393-404.

7. O'Hare A, Fenlon H. Virtual colonoscopy in the detection of colonic polyps and neoplasms. Best Pract Res Clin Gastroenterol. 2006 Feb;20(1):79-92.

8. Kronborg O, Fenger C. Clinical evidence for the adenomacarcinoma sequence. Eur J Cancer Prev. 1999 Dec;8 Suppl 1:S73-86.

9. Miller KM, Waye JD. Approach to colon polyps in the elderly. Am J Gastroenterol. 2000 May;95(5):1147-51.

10. Pickhardt PJ. Differential diagnosis of polypoid lesions seen at CT colonography (virtual colonoscopy). Radiographics. 2004 Nov-Dec;24(6):1535-56; discussion 57-9.

11. Manual de Terapêutica - Cirurgia. Associação Catarinense de Medicina. Florianópolis: Nova Letra 2006:205-08.

12. Santos JM dos, Felício F, Oliveira JCC de, Silva CA, Schneider EJ. Tratamento endoscópico dos pólipos colorretais. Arq Cat Med. 1996;25(3):230-5.

13. Winawer SJ, Zauber AG, Ho MN, O'Brien MJ, Gottlieb LS, Sternberg SS, et al. Prevention of colorectal cancer by colonoscopic polypectomy. The National Polyp Study Workgroup. N Engl J Med. 1993 Dec 30;329(27):1977-81.

14. Schulmann K, Reiser M, Schmiegel W. Colonic cancer and polyps. Best Pract Res Clin Gastroenterol. 2002 Feb;16(1):91114.

15. Giacosa A, Frascio F, Munizzi F. Epidemiology of colorectal polyps. Tech Coloproctol. 2004 Dec;8 Suppl 2:s243-7.

16. Fukami N, Lee JH. Endoscopic treatment of large sessile and flat colorectal lesions. Curr Opin Gastroenterol. 2006 Jan;22(1):54-9.

17. Bonadeo NM, Atolini Jr N, Foiatto JC, Lovison AF, Saleh CS, Saleh JNS. Prevalência de pólipos com displasia de alto grau em pacientes com idade inferior a 50 anos Gastroenterol Endosc Dig. 2007 Jan/Fev;26(1):5-7.

18. Markowitz AJ, Winawer SJ. Management of colorectal polyps. CA Cancer J Clin. 1997 Mar-Apr;47(2):93-112.

19. Loeve F, van Ballegooijen M, Snel P, Habbema JD. Colorectal cancer risk after colonoscopic polypectomy: a populationbased study and literature search. Eur J Cancer. 2005 Feb;41(3):416-22.

20. Church JM. Experience in the endoscopic management of large colonic polyps. ANZ J Surg. 2003 Dec;73(12):988-95.
21. Cummings OW. Pathology of the adenoma-carcinoma sequence: from aberrant crypt focus to invasive carcinoma. Semin Gastrointest Dis. 2000 Oct;11(4):229-37.

22. Mitros FA. Polyps: the pathologist's perspective. Semin Surg Oncol. 1995 Nov-Dec;11(6):379-85.

23. Itzkowitz SH. Gastrointestinal adenomatous polyps. Semin Gastrointest Dis. 1996 Apr;7(2):105-16.

24. Bond JH. Interference with the adenoma-carcinoma sequence. Eur J Cancer. 1995 Jul-Aug;31A(7-8):1115-7.

25. Seitz U, Bohnacker S, Seewald S, Thonke F, Brand B, Braiutigam $\mathrm{T}$, et al. Is endoscopic polypectomy an adequate therapy for malignant colorectal adenomas? Presentation of 114 patients and review of the literature. Dis Colon Rectum. 2004 Nov;47(11):1789-96; discussion 96-7.

26. Huang CS, O'Brien M J, Yang S, Farraye FA. Hyperplastic polyps, serrated adenomas, and the serrated polyp neoplasia pathway. Am J Gastroenterol. 2004 Nov;99(11):2242-55.

27. Higuchi T, Jass JR. My approach to serrated polyps of the colorectum. J Clin Pathol. 2004 Jul;57(7):682-6.

28. Snover DC, Jass JR, Fenoglio-Preiser C, Batts KP. Serrated polyps of the large intestine: a morphologic and molecular review of an evolving concept. Am J Clin Pathol. 2005 Sep;124(3):380-91.

29. Watne AL. Colon polyps. J Surg Oncol. 1997 Nov;66(3):207-14.

30. Beck DE, Opelka FG, Hicks TC, Timmcke AE, Khoury DA, Gathright JB, Jr. Colonoscopic follow-up of adenomas and colorectal cancer. South Med J. 1995 May;88(5):567-70.

31. Halligan S, Altman DG, Taylor SA, Mallett S, Deeks JJ, Bartram CI, et al. CT colonography in the detection of colorectal polyps and cancer: systematic review, meta-analysis, and proposed minimum data set for study level reporting. Radiology. 2005 Dec;237(3):893-904.

32. Villavicencio RT, Rex DK. Colonic adenomas: prevalence and incidence rates, growth rates, and miss rates at colonoscopy. Semin Gastrointest Dis. 2000 Oct;11(4):185-93.

33. Repici A, Tricerri R. Endoscopic polypectomy: techniques, complications and follow-up. Tech Coloproctol. $2004 \mathrm{Dec} ; 8$ Suppl 2:s283-90.

\section{Endereço para correspondência:}

JOSÉ MAURO DOS SANTOS

R. Presidente Coutinho, 579 sala 201 - B

Florianópolis - SC

CEP: $88015-231$

Tel: (48) 3223-6069

E-mail: proctoclinica_fpolis@yahoo.com.br 